\title{
Orbital Approach to High Temperature Superconductivity
}

\section{Peter Love}

Department of Chemistry, University of Connecticut at Stamford, University Place, Stamford, CT, USA

Correspondence to: Peter Love, peter.love@uconn.edu

Keywords: High Temperature Superconductivity, D-Orbital Capture, Cuprates, Coordinate Covalent Bonding

Received: November 27, $2018 \quad$ Accepted: January 14, $2019 \quad$ Published: January 17, 2019

Copyright $\odot 2019$ by authors and Scientific Research Publishing Inc.

This work is licensed under the Creative Commons Attribution International License (CC BY 4.0).

http://creativecommons.org/licenses/by/4.0/

\section{Open Access}

\section{ABSTRACT}

High temperature superconductivity in cuprates is explained in terms of 3d-orbital capture in copper. In elemental $\mathrm{Cu} 3 \mathrm{~d}$-orbital capture abstracts an electron from the $4 \mathrm{~s}^{2}$ valence orbital, and leaves it as $4 s^{1}$. This is known since $\mathrm{Cu}$ occurs in Group IB of the Periodic Table. This forms an electron vacancy, or hole, in the valence shell. Therefore, the energy of $3 \mathrm{~d}$-orbital capture is stronger than the energy of unpairing of a paired-spin $4 \mathrm{~s}^{2}$ orbital. In cuprates $3 \mathrm{~d}$-orbital capture abstracts an electron from a $\mathrm{Cu}-\mathrm{O}$ covalent bond, and leaves a hole in the excited state orbital. By electron-hole migration the excited state orbital leads to a coordinate covalent bond. This leads to superconductivity. The $3 \mathrm{~d}$-orbital process accounts for superconductivity and insulator behavior in cuprates. These results lend credence to the statement that $3 \mathrm{~d}$-orbital capture in copper is the cause of high temperature superconductivity.

\section{INTRODUCTION}

High temperature superconductivity (HTS) was discovered in 1986 by Bednorz and Müller [1]. Since then thousands of papers and more than four monographs have been written on this subject in attempts to explain the origin and nature of the phenomenon. Within the approximately thirty year period since then no mathematical theories have explained, or related, HTS to specific atoms in the Periodic Table. The origin of HTS, or why it occurs specifically in cuprates, has not been given.

This paper is an elementary verbal explanation of how and why HTS occurs in cuprates. It is a statement that HTS in cuprates is the result of the $3 \mathrm{~d}$-orbital capture process in copper. Some properties of typical HTS materials reported in the literature that support this premise are given. High temperature superconductivity can be understood verbally without appeal to abstract theories, or mathematical formalism.

Superconductivity is often defined as the temperature, $T_{\mathcal{o}}$ at which the electrical resistance of a conductor goes to zero absolute temperature. A superconductor also exhibits the electrical property of persistent currents [2]. This is the property where an induced electric current formed in a circular superconductor wire continues indefinitely without attenuation. This implies that the superconducting electrons 
are suspended in free space by electrostatic fields. They do not physically come in contact with their atomic environment. A chemical, or any other, explanation of superconductivity should account for this property. The coordinate covalent bond (CCB) model discussed herein is consistent with the property of persistent currents.

\section{Coordinate Covalent Bond Formation and Superconductivity}

It has been proposed that a classical coordinate covalent bond, $\mathrm{CCB}$, is the basic bonding responsible for superconductivity [3]. A CCB contains 2 paired electron spins (a Lewis pair) supported by an electron pair donor (EPD) species, and an electron pair acceptor (EPA) species. When the residual electrostatic attraction of the EPD species equals the residual electrostatic attraction of the EPA species the bonded Lewis pair is effectively suspended in free space.

The EPD species often are Group IIA, or Group B, elements that have classical $\mathbf{n} \mathbf{s}^{2}$ valence shell electrons available. The EPA species sometimes are diffuse and weak orbital sites. This is true for cuprate materials.

\section{THEORY}

\subsection{Electronic Structure of Copper}

Since high temperature superconductivity with transition temperatures, $T_{c} \geq 90 \mathrm{~K}$, has only been found in cuprates one expects that the electron configuration of copper is of primary concern in this process.

In the Periodic Table the electron filling order of $4^{\text {th }}$ Period B Group elements is: $\left[\mathrm{Ar}^{18}\right] 4 \mathrm{~s}^{2} 3 \mathrm{~d}^{x}$. Here, $x=1-10$, for elements with atomic numbers, $21-30$. The A-B group designation in the Periodic Table is used herein. This group designation provides more information about element electronic structure than the currently accepted IUPAC 1 - 18 group designation version.

The electronic structure of copper is very well known. It is given here since it is the basis of this discussion. The electron configuration of copper in terms of filling order should be,

$$
\mathrm{Cu}:\left[\mathrm{Ar}^{18}\right] 4 \mathrm{~s}^{2} 3 \mathrm{~d}^{9}
$$

Many chemical reactions of this element do not correspond to a $4 \mathrm{~s}^{2}$ valence shell. The observed valence shell configuration is $4 \mathrm{~s}^{1}$. Abstraction of an electron from a $4 \mathrm{~s}^{2}$ orbital to complete the $3 \mathrm{~d}$-shell leads to a dynamic equilibrium between the configurations: $4 \mathrm{~s}^{2}+3 \mathrm{~d}^{9}$, and $4 \mathrm{~s}^{1}+3 \mathrm{~d}^{10}$. This is shown in Equation (2).

$$
4 \mathrm{~s}^{2}+3 \mathrm{~d}^{9} \rightleftarrows 4 \mathrm{~s}^{1}+3 \mathrm{~d}^{10}
$$

The equilibrium in Equation (2) is shifted to the right since copper is in Group IB, and forms many monovalent salts.

\subsection{3d-Orbital Capture}

The process of 3d-orbital capture is expressed by the equilibrium in Equation (2). Complete filling of the $3 \mathrm{~d}$-shell leaves one electron and an electron deficiency in the valence shell. This makes this valence shell an electron acceptor. In solid state physics parlance $3 \mathrm{~d}$-orbital capture leaves a "hole" in the valence shell of copper. Electrons occur in stable atoms and molecules as paired electron orbitals. A "hole" is a vacancy in a normal two electron orbital. In the orbital shift process the $\mathrm{Cu}$ atom retains an electrostatically neutral atomic environment. There is no formal charge transfer external to the copper atom in the transition.

The process of 3d-orbital capture is expected to be essentially independent of ambient lattice temperature. It occurs at the Fermi level, $\mathscr{E}_{F}$. For $\mathrm{Cu}, \mathscr{E}_{F}=7.00 \mathrm{eV}$ [4]. Ambient lattice temperature, $\sim 25^{\circ} \mathrm{C}$, corresponds to, $\mathscr{E}=\sim 0.026 \mathrm{eV}$. (This follows from the definition of the Boltzmann constant, 
$k=8.617 \times 10^{-5} \mathrm{eV} \cdot \mathrm{K}^{-1}$ ). The value, $\mathscr{E}=0.026 \mathrm{eV}$, is negligible as compared to $\mathscr{E}_{F}$ for $\mathrm{Cu}$.

The reaction of $3 \mathrm{~d}$-orbital capture is the cause, or origin, of HTS. It is the source of energy that leads to hole formation in cuprates, and subsequently to boson state population, and superconductivity. The energy of $3 \mathrm{~d}$-orbital capture in $\mathrm{Cu}$ is stronger than the energy of unpairing of the expected $4 \mathrm{~s}^{2}$ valence shell orbital of this element. This obviously is true since the ground state valence shell configuration is $4 s^{1}$.

It is the neutral 29 electron copper atom that engages in 3d-orbital capture. Removal of the valence shell electrons of copper eliminates the equilibrium in Equation (2). Therefore, $\mathrm{Cu}^{2+}$ cannot engage in the $3 \mathrm{~d}$-orbital capture process.

Discussions of $\mathrm{Cu}^{2+}$ in describing the lattice structure and electron orbitals of cuprates are appropriate. For example, Saxena [5] concludes that in cuprates since oxygen "favors" $\mathrm{O}^{2-}$, copper must be, $\mathrm{Cu}^{2+}$. This is reasonable since cuprates are refractory materials that are synthesized at temperatures $\leq 1000 \mathrm{~K}$. An excellent discussion of cuprates, and the synthesis of bulk and thin films of these materials is given by Saxena [6].

However, there must be a degree of covalency in some $\mathrm{Cu}-\mathrm{O}$ bonds in order for 3d-orbital capture to occur in cuprates. Saxena [7] states that NMR experiments show evidence that oxygen $p \pi$-hybridized orbitals are involved in conduction planes in cuprates. These are the type of covalent bonds that can contribute to hole formation.

\section{RESULTS}

\subsection{Occurrence of High Temperature Superconductivity}

One of the earliest HTS cuprates found was a macromolecular solid that has the empirical formula, $\mathrm{YBa}_{2} \mathrm{Cu}_{3} \mathrm{O}_{7}$. This has, $T_{c}=90 \mathrm{~K}$. [8]. For the sake of the discussion here we consider a cuprate to be a macromolecular solid that contains the $\mathrm{Cu}_{3} \mathrm{O}_{7}^{7-}$ anion. A number of cuprate materials with various empirical formuli are known [9]. Some of these are reported to have $T_{c}$ values as high as $125 \mathrm{~K}$. These cuprate materials in general are expected to have the same $3 \mathrm{~d}$-orbital capture process in common.

\subsection{Possible Reaction Sequence for Formation of Coordinate Covalent Bonds}

The 3d-orbital capture process in cuprates is essentially the same as that which occurs in elemental $\mathrm{Cu}$. As stated in Section 2.2 in elemental $\mathrm{Cu}$ 3d-orbital capture leaves one electron and a hole in the valence shell. In cuprates $3 \mathrm{~d}$-orbital capture abstracts an electron from a covalently bonded $\mathrm{Cu}-\mathrm{O}$ orbital in $\mathrm{Cu}_{3} \mathrm{O}_{7}^{7-}$, and leaves a hole in this orbital. This is shown by Equation (3).

$$
\mathrm{Cu}-\mathrm{O}(\text { neutral })+3 \mathrm{~d}^{9} \rightleftarrows \mathrm{Cu}-\mathrm{O}(\text { with } 1 \text { hole })+3 \mathrm{~d}^{10}
$$

This disrupts the $\mathrm{Cu}-\mathrm{O}$ orbital and leaves one electron and an electron vacancy, or "hole", in an excited state in this orbital. This is expected to be a random reversible excited state process. This is indicated to be true as is shown in Section 4.1 in Figure 2 by the broadened heat capacity anomalies of several cuprates.

The high $T_{c}$ values of cuprates are the result of the high concentrations, or population, of the excited state covalent $\mathrm{Cu}-\mathrm{O}$ orbitals that are formed by electron abstraction via the continuous $3 \mathrm{~d}$-orbital capture process. The continuous $3 \mathrm{~d}$-orbital capture process is the cause of the sustained high "hole" concentrations in these orbitals.

In the 3d-orbital capture process, $1 \mathrm{Cu}$ atom $\rightarrow 1 \mathrm{Cu}-\mathrm{O}$ orbital (with 1 hole). An EPA site is a 2 electron acceptor orbital, i.e., a 2 hole orbital. There is a stoichiometry matching problem here. In principle two 1 hole orbitals can combine, or rehybridize, to form a vacant electron pair acceptor, EPA, orbital. This is shown symbolically in Equation (4).

$$
2 \mathrm{Cu}-\mathrm{O}(\text { with } 1 \text { hole }) \rightleftarrows \text { EPA orbital(with } 2 \text { holes) }
$$

This process is sometimes referred to as "electron-hole migration". This type of electron-hole migration is known to occur [10]. It is necessary to form a coordinate covalent bond. These are expected to be 
disordered processes. Again, this effect is shown by the broadened heat capacity anomaly curves that are shown in Section 4.1.

Equation (4) represents the same process that is described by Miller [11]. The primary difference is that Miller uses the physicist's term "Cooper pair" in place of "electron pair", or the chemist's "Lewis pair" description.

\subsection{Orbital Hybridization of Copper in the Crystal Lattice}

It is known that the cuprate crystal lattice has a perovskite structure [12]. This is important, and is generally given in discussions of cuprate superconductivity. Equally important, if not more so, is the orbital hybridization about copper in the crystal lattice. There is little, if any, discussion of this in the cuprate literature. It is the orbital hybridization of copper that leads to boson formation and superconductivity.

Cuprates are known to be planar superconductors [7]. One expects that orbital hybridization of an atom determines the geometric structure of orbitals about that species. This is not necessarily true for extended solid state lattices synthesized at pyrolytic temperatures. Orbital hybridization about the $\mathrm{Cu}$ central atom of attachment that is involved in planar mobile electron transport in complexes is expected to be $\mathrm{dsp} \mathrm{p}^{2}$. This hybridization results in square planar orbital geometry $[13,14]$.

In elemental $\mathrm{Cu} 3 \mathrm{~d}$-orbital capture occurs on a single $\mathrm{Cu}$ atom. This is a highly localized short range process. This suggests that $3 \mathrm{~d}$-orbital capture in cuprates occurs on $\mathrm{Cu}$ atoms where the four hybridized $\mathrm{dsp}^{2}$ orbital lobes are on the same $\mathrm{Cu}$ atom. For copper in a cuprate the hybridized orbitals are expected to result from a $3 \mathrm{~d}$-orbital on $\mathrm{Cu}$, one $4 \mathrm{~s}$-orbital on $\mathrm{Cu}$, and two p-orbitals from $\mathrm{Cu}-\mathrm{O}$ covalent bonds attached to this $\mathrm{Cu}$ atom. There are 2 isomeric $\mathrm{dsp}^{2}$ structures possible. Opposite and adjacent positions of groups in the hybridized orbitals leads to linear chain and square planar configurations, respectively.

The atom stoichiometry of this planar hybridized entity is $\mathrm{CuO}_{2}$. This implies that the species responsible for electron delocalization in cuprates has the stoichiometry $\mathrm{CuO}_{2}$. The basic delocalized electron carrier is expected to be a covalently bonded $\mathrm{CuO}_{2}$ unit. This is consistent with the observation of Alecu [15] that the more layers of " $\mathrm{CuO}_{2}$ " per unit volume there are in a cuprate crystal structure, the higher is the value of $T_{c}$

Groups of correlated parallel CCBs arrayed as an effective EPA Bravais lattice [16] in a crystal structure provide channels for delocalization of Lewis pairs as boson wave functions in planar sheets. These will have adjacent EPD groups and adjacent EPA sites in the crystal lattice. The effective Bravais lattice is subject to the atomic vibrations of the crystal lattice. It is these atomic vibrations that determine the transition temperature, $T_{c}$ The value of $T_{c}$ is also the temperature above which superconductivity does not occur. When, $T>T_{\mathcal{o}}$ lattice vibrations can be large enough to prevent the existence of an effective extended Bravais lattice. This results in $T_{c}$ being dependent on the ambient lattice temperature.

\section{EXPERIMENTAL VERIFICATION}

\subsection{Line Shapes of Fermion-Boson Transition Heat Capacity Anomalies}

Verification of delocalized 3d-orbital capture in cuprates is shown by comparison of the fermion-boson transition heat capacity anomalies of low and high temperature superconductors. Low temperature superconductors with, $T_{c} \leq 10 \mathrm{~K}$, typically have very sharp narrow vertical transitions. This is indicative of very weak essentially singular EPA sites in the elemental materials. An example is shown in Figure 1 for niobium, $\mathrm{Nb}$, with, $T_{c}=9.26 \mathrm{~K}$. This element has the highest $T_{c}$ value of the superconducting elements in the Periodic Table.

High temperature cuprates have broadened heat capacity anomalies. Examples are, $\mathrm{YBa}_{2} \mathrm{Cu}_{3} \mathrm{O}_{7-\delta}, T_{c}=$ $\sim 92 \mathrm{~K}$, and $\mathrm{DyBa}_{2} \mathrm{Cu}_{3} \mathrm{O}_{7-\delta}, T_{c}=\sim 92 \mathrm{~K}$. Heat capacity anomalies for these non-stoichiometric materials are shown in Figure 2.

The lack of a sharp vertical transition and broadened temperature ranges of the vertical transition regions of the heat capacity anomalies are clear indications of multiple 3d-orbital capture electron abstraction reactions. 


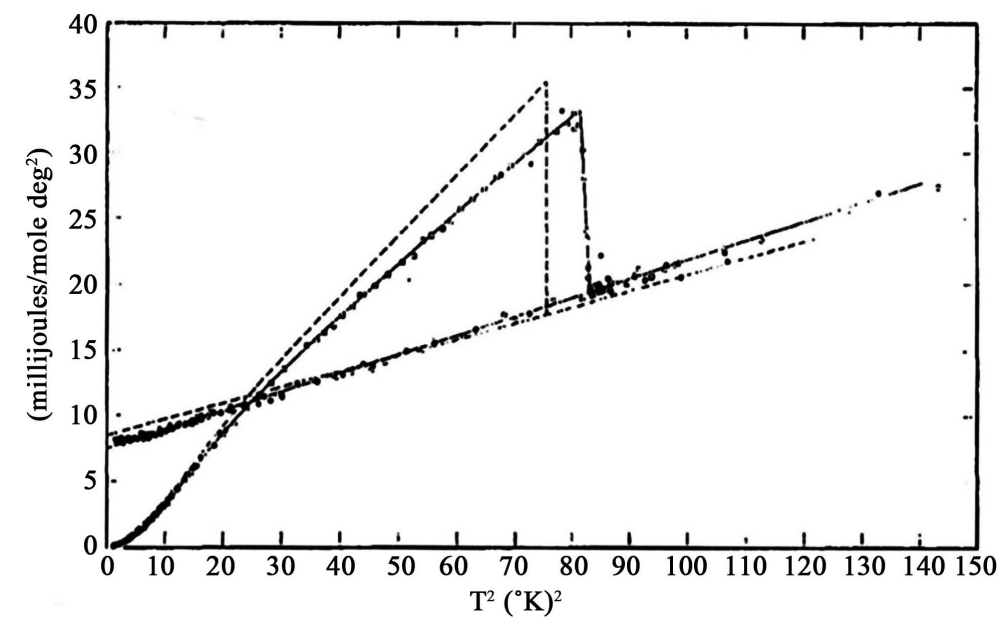

Figure 1. Niobium heat capacity anomaly for, (a) initial sample (- - - ); and (b) after $2623 \mathrm{~K}$ heat treatment (- ) [19].

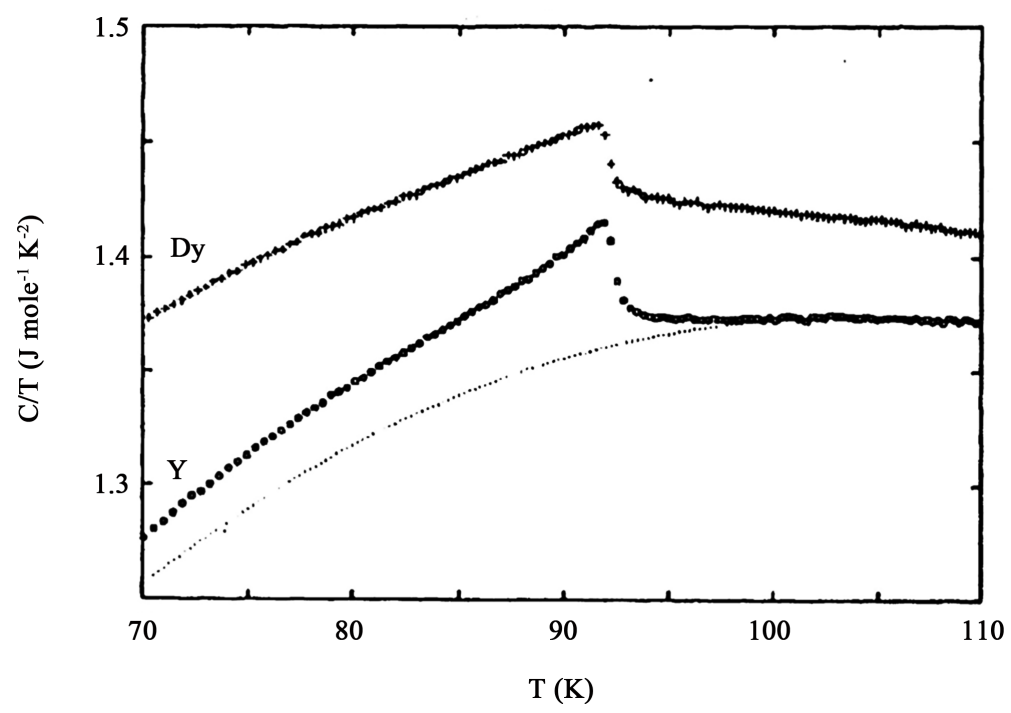

Figure 2. Heat capacity anomalies of $\mathrm{DyBa}_{2} \mathrm{Cu}_{3} \mathrm{O}_{7-\delta}$, and $\mathrm{YBa}_{2} \mathrm{Cu}_{3} \mathrm{O}_{7-\delta}[20]$.

\subsection{Variation of $T_{c}$ with Oxygen Stoichiometry of Cuprates}

Saxena has reported that $T_{c}$ is a complex and sensitive function of the oxygen stoichiometry in $\mathrm{YBa}_{2} \mathrm{Cu}_{3} \mathrm{O}_{7-\delta}$ [17]. For example, when $\delta<0.2, T_{c}=\sim 92 \mathrm{~K}$. When $\delta=0.4, T_{c}=\sim 60 \mathrm{~K}$. When $\delta=1.0$, the stoichiometry is, $\mathrm{YBa}_{2} \mathrm{Cu}_{3} \mathrm{O}_{6}$. This material is not a superconductor. The unit cell parameter is also varied.

The formula, $\mathrm{YBa}_{2} \mathrm{Cu}_{3} \mathrm{O}_{6}$, corresponds to an ionic crystal structure that appears to contain only $\mathrm{Cu}^{2+}$. As stated in Section 2.2 a crystal lattice that contains only $\mathrm{Cu}^{2+}$ ions cannot support superconductivity. The fact that cuprate crystal structures that appear to contain only $\mathrm{Cu}^{2+}$ ions are not superconducting supports the thesis that $3 \mathrm{~d}$-orbital capture is the cause of HTS.

\section{DISCUSSION}

\subsection{3d-Orbital Capture as Cause of HTS}

The Group I B elements, $\mathrm{Cu}, \mathrm{Ag}$ and $\mathrm{Au}$, are the only elements in which d-orbital capture occurs. Of these copper is the only element that supports low energy d-orbital capture equilibria. Facile $3 \mathrm{~d}$-orbital 
capture is unique for $\mathrm{Cu}$. That cuprates contain copper is the reason that HTS only occurs in these materials.

Since elemental $\mathrm{Cu}$ has only one electron in the valence shell it cannot support superconductivity [3]. The 3d-orbital capture process that prevents the occurrence of superconductivity in this element is the cause of high temperature superconductivity in cuprates.

\subsection{Holes in the Conduction Plane}

Saxena [5] and Ford and Saunders [18] discuss hole carriers, and possible sources of holes, in $\mathrm{Cu}-\mathrm{O}$ conduction planes in cuprates. Several specific atomic orbitals, and holes in the conduction plane, are discussed by Saxena [7]. Saxena states that "conduction oxygen holes" in the $p \pi$ orbitals are responsible for cuprate superconductivity. This type of orbital is expected to be involved in $3 \mathrm{~d}$-orbital capture.

These authors do not mention, or discuss 3d-orbital capture as a source of holes, or a source of energy, for HTS. Holes in the conduction plane are certainly necessary for HTS. However, holes are simply a means to an end. As stated in Section 2.2 a "hole" is an electron deficiency, i.e., vacancy, in an orbital. It is the electron deficiencies in covalent C-O bonds that are necessary for the formation of correlated CCB's that form the electrostatic channels for suspension of itinerate superconducting electrons.

It is reported that $\mathrm{La}_{2} \mathrm{CuO}_{4}$ is not superconducting [18]. If this material contains only ionic $\mathrm{Cu}^{2+}$ then this is understandable. This implies that there no covalent $\mathrm{Cu}-\mathrm{O}$ bonds present to allow $3 \mathrm{~d}$-orbital capture to occur. The material, $\mathrm{La}_{2-\mathrm{x}} \mathrm{Ba}_{\mathrm{x}} \mathrm{CuO}_{4}$, is a superconductor when $x \geq 0.06$ [18]. The effective lattice structure is reported to be changed upon Ba doping. This can be expected since the total number of charged cations is now changed. This can allow covalent $\mathrm{Cu}-\mathrm{O}$ bonds to occur. This facilitates $3 \mathrm{~d}$-orbital capture, and leads to superconductivity.

\section{SUMMARY}

The $T_{c}$ values of cuprate HTS materials are affected by many factors. Of these, $3 \mathrm{~d}$-orbital capture in $\mathrm{Cu}$ appears to be the primary cause for the occurrence of high $T_{c}$ values in cuprates. The fact that cuprates contain $\mathrm{Cu}$ is the obvious reason that these are the only materials found that support HTS where, $T_{c} \geq 90$ $\mathrm{K}$. Atom stoichiometry of the material, crystal structure, and the dsp ${ }^{2}$ hybridization state of $\mathrm{Cu}$ are important, and maybe related. The basic entity involved in mobile electron transport is expected to be covalently bonded $\mathrm{CuO}_{2}$. Recognition of the presence and efficacy of 3d-orbital capture in copper is the key to understanding high temperature superconductivity in cuprates.

\section{ORAL PRESENTATION}

This paper was given as an oral presentation at the 254th Meeting of the American Chemical Society, Washington, D.C., August, 20-24, 2017, Physical Chemistry Division.

\section{RESEARCH FUNDING}

This research did not receive any specific grant from funding agencies in the public, commercial, or not-for-profit sectors.

\section{CONFLICTS OF INTEREST}

There are no competing interests.

\section{REFERENCES}

1. Bednorz, J.G. and Müller, K.A. (1986) Possible High $T_{c}$ Superconductivity in the Ba-La-Cu-O System. Zeitschrift für Physik B Condensed Matter, 64, 189-193. https://doi.org/10.1007/BF01303701

2. Ashcroft, N.W. and Mermin, N.D. (1976) Solid State Physics. Saunders, Philadelphia, 728-730. 
3. Love, P. (2012) Chemical Bonding and Thermodynamics in Superconductivity and Superfluidity. International Journal of Thermophysics, 33, 795-820. https://doi.org/10.1007/s10765-012-1188-y

4. Ashcroft, N.W. and Mermin, N.D. (1976) Solid State Physics. Saunders, Philadelphia, 38-39.

5. Saxena, A.K. (2012) High-Temperature Superconductors, 2nd Edition, Springer, Heidelberg, 132-134. https://doi.org/10.1007/978-3-642-28481-6

6. Saxena, A.K. (2012) High-Temperature Superconductors. 2nd Edition, Springer, Heielberg, 101-107. https://doi.org/10.1007/978-3-642-28481-6

7. Saxena, A.K. (2012) High-Temperature Superconductors. 2nd Edition, Springer, Heidelberg, 139. https://doi.org/10.1007/978-3-642-28481-6

8. Saxena, A.K. (2012) High-Temperature Superconductors. 2nd Edition, Springer, Heidelberg, 8. https://doi.org/10.1007/978-3-642-28481-6

9. Saxena, A.K. (2012) High-Temperature Superconductors. Springer, Heidelberg, 51. https://doi.org/10.1007/978-3-642-28481-6

10. Stranks, S.D., Giles, E.E., Grancini, G., Menelaou, C., Alcocer, M.J.P., Leijtens, T., et al. (2013) Electron-Hole Diffision Lengths Exceeding 1 Micrometer in an Organometal Trihalide Perovskite Absorber. Science, 342, 341-344. https://doi.org/10.1126/science.1243982

11. Miller, J. (2013) Ultrasound Measurements Reveal a Long-Sought Phase Transition in Superconducting Cuprates. Physics Today, 66, 12-15. https://doi.org/10.1063/PT.3.2066

12. Saxena, A.K. (2012) High-Temperature Superconductors. 2nd Edition, Springer, Heidelberg, 43-49. https://doi.org/10.1007/978-3-642-28481-6_2

13. Douglas, B.E., McDaniel, D.H. and Alexander, J.J. (1983) Concepts and Models of Inorganic Chemistry. 2nd Edition, Wiley, New York, 58.

14. Durrant, P.J. and Durrant, B. (1962) Introduction to Advanced Inorganic Chemistry. Wiley, New York, 151-152.

15. Alecu, G. (2004) Crystal Structures of Some High Temperature Suprconductors. Romanian Reports in Physics, 56, 404-412.

16. Ashcroft, N.W. and Mermin, N.D. (1976) Solid State Physics. Saunders, Philadelphia, 64-66.

17. Saxena, A.K. (2012) High-Temperature Superconductors. 2nd Edition, Springer, Heidelberg, 46-47. https://doi.org/10.1007/978-3-642-28481-6

18. Ford, P.J., Saunders, G.A. (2005) The Rise of the Superconductors. CRC Press, Boca Raton, 50-53.

19. Hirshfeld, A.T., Leupold, H.A. and Boorse, H.A. (1962) Superconducting and Normal Specific Heat of Niobium. Physical Review, 127, 1501-1507. https://doi.org/10.1103/PhysRev.127.1501

20. Schnelle, W., Braun, E., Broicher, H., Weiss, H., Geus, H., Ruppel, S., et al. (1989) Superconducting Fluctuations in $\mathrm{Bi}_{2} \mathrm{Sr}_{2} \mathrm{Ca}_{2} \mathrm{Cu}_{3} \mathrm{O}_{\mathrm{x}}$. Physica C, 161, 123-135. https://doi.org/10.1016/0921-4534(89)90120-2 